\title{
O IMPACTO DA IMPLEMENTAÇÃO DA TECNOLOGIA BIM NO PROCESSO CONSTRUTIVO: ESTUDO DE CASO DE RESIDÊNCIA DO ANO DE 1960 RN - BRASIL
}

\author{
Josyanne Giesta(1), Mateus Silva(2), Aline Coelho ${ }^{(1)}$ \\ (1) Instituto Federal de Educação, Ciência e Tecnologia do Rio Grande do Norte, Natal \\ (2) Centro Universitário Facex, Natal
}

\begin{abstract}
Resumo
As constantes transformações que o mercado da construção civil vem sofrendo proporcionam um ambiente em que os antigos métodos construtivos estão se tornando obsoletos, diante disso o Building Information Modeling (BIM) se apresenta como um mecanismo capaz de intensificar a racionalização do fluxo de trabalho utilizado na área de Arquitetura, Engenharia e Construção (AEC), promovendo através da interoperabilidade dos softwares uma maior segurança e precisão nos processos de quantificação e controle de tempo estabelecidos no cronograma do empreendimento. O presente trabalho tem como objetivo mostrar a viabilidade da implementação da tecnologia BIM ao processo da obra como um todo. A metodologia utilizada foi dividida nas seguintes fases: I- Contexto teórico, quantificação e planejamento; IIUtilização de softwares BIM; III- Estudo de caso aplicado ao processo construtivo de demolição e reforma de uma residência do ano de 1960. O resultado permite visualizar que a utilização de ferramentas da tecnologia BIM possibilitou maior otimização e segurança nos processos de extração de quantitativos e controle da visualização das fases da obra, constatando a viabilidade da implementação no processo construtivo.
\end{abstract}

\section{Introdução}

Muito se discute que os avanços tecnológicos são as ferramentas que apresentam o potencial necessário para mudar a perspectiva da construção civil. O Building Information Modeling (BIM) é um recurso da área de Arquitetura, Engenharia e Construção (AEC) que propõe a criação de um modelo virtual da edificação capaz de conter uma geometria que fornece informações das etapas construtivas, como os insumos necessários para a realização do projeto. Estudos nacionais e internacionais revelam que a produtividade das empresas do setor da construção é consideravelmente mais baixa do que a do resto da economia [1].

A tecnologia BIM promove uma colaboração mais eficiente entre todas as partes interessadas do projeto durante o ciclo de vida do empreendimento, o que proporciona mais resultado na entrega do produto final [2]. 
No Brasil o decreto $\mathrm{n}^{\circ} 9.983$, de 22 de agosto de 2019 se apresenta como documento que dispõe sobre a estratégia nacional de disseminação do BIM em todo país. Dentre os objetivos no processo de difusão podem se destacar o desenvolvimento de normas técnicas, guias e protocolos específicos para sua adoção, criação de condições favoráveis para o investimento público e privado, e incentivar a concorrência no mercado por meio de padrões neutros de interoperabilidade BIM [3].

Recentemente, através do decreto $\mathrm{n}^{\circ} 10.306$ de 02 de abril de 2020, foram reforçadas as iniciativas nesse sentido, quando foi estabelecido a utilização do BIM na execução direta ou indireta de obras e serviços de engenharia realizada por órgãos e pelas entidades da administração pública federal [4].

A quarta dimensão do BIM (BIM 4D) permite que se realize uma análise de monitoramento aos tempos de construção, dessa forma a implementação desse fator a modelagem 3D proporciona a produção de um cronograma de controle do modelo.

De acordo com Lino, Lacerda, Reis e Caires, o uso de ferramentas de gestão e planejamento apoiadas em modelo BIM está se tornando um procedimento essencial nas empresas de construção, pois seu uso pode potencializar uma diminuição de risco na empreitada assim como também permite de forma pratica criar cenários de simulação do planejamento da construção [5].

Vale salientar ainda a existência da dimensão 5D a qual corresponde a uma evolução da dimensão 4D através da adição da variável custo, tornando-se possível prever e controlar os custos em todas as fases de construção. Com a evolução do modelo as estimativas de custo são melhoradas consoante ao aumento de detalhamento, e é possível medir o desempenho financeiro do estado atual da construção através das análises extraídas do modelo 5D, os autores acrescentam que de forma sucinta esta dimensão "procura extrair os quantitativos para execução dos elementos", segundo Coelho, Couto, Leitão, e Morgado [6].

Apesar dos grandes avanços conquistados a tecnologia BIM ainda enfrenta inúmeras adversidades de consolidação tanto em âmbito acadêmico quanto no próprio mercado da construção civil, diante disso é extremamente necessário o desenvolvimento de estudos que evidenciem os benefícios e as próprias dificuldades dos usos de softwares que trabalhem com essa tecnologia, seja com objetivo de extração de quantitativos ou mesmo no planejamento das fases da construção.

O presente artigo apresenta um estudo de caso de uma residência do ano de 1960 na cidade de Tibau do Sul, no Rio Grande do Norte, Brasil, e o balanço da utilização da metodologia de trabalho BIM na extração de quantitativos e no planejamento da construção da residência. Com os resultados gerados planeja-se obter informações de como a metodologia empregada pode melhorar o fluxo de trabalho durante o processo construtivo.

\section{Metodologia}

A metodologia utilizada para o desenvolvimento desse trabalho foi dividida em três fases representadas na Figura 1, que descreve: Contextualização teórica, utilização dos softwares, e estudo de aplicação efetiva em uma edificação real. Esse fluxo de trabalho permitiu um bom desenvolvimento do artigo aqui apresentado. 


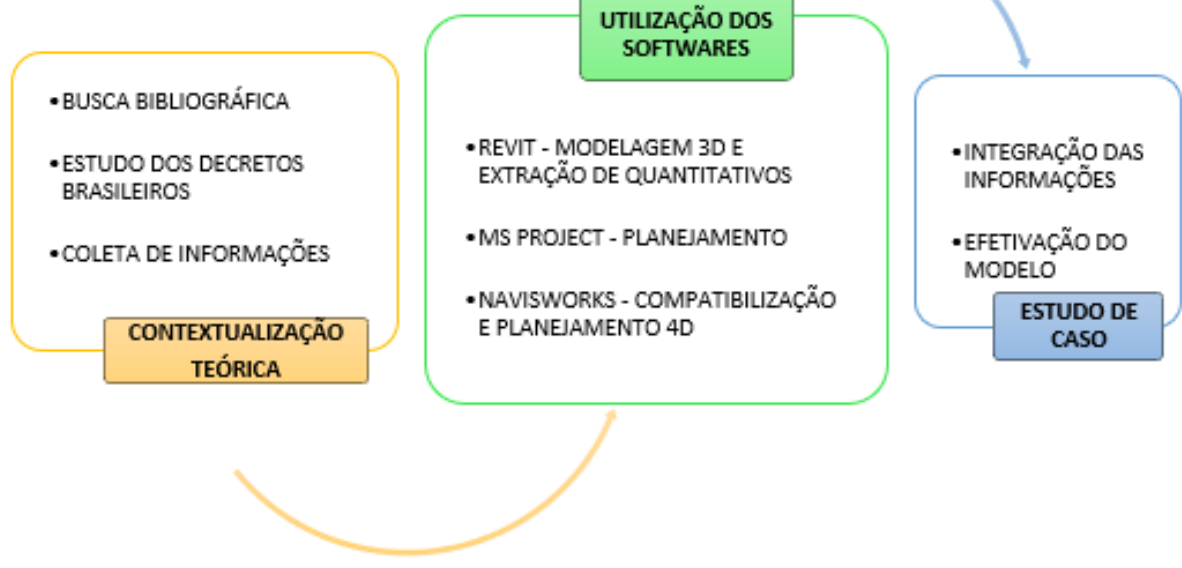

Figura 1: Fluxograma das fases.

A contextualização teórica partiu da pesquisa bibliográfica de trabalhos contidos nas atas do PTBIM/2016, PTBIM/2018, e nos decretos já lançados pelo governo brasileiro, com o objetivo de reunir informações que pudessem proporcionar um estudo com bom embasamento teórico. Atenta-se para o fato que os artigos escolhidos são de trabalhos que remetem a propostas equivalentes as presente nesse estudo.

$\mathrm{Na}$ fase de utilização de softwares foram utilizados o Autodesk Revit na etapa de modelagem 3D e extração de quantitativos, o Microsoft Project para produção da Estrutura Analítica do Projeto (EAP), e posteriormente o Autodesk Navisworks para elaboração da compatibilização e planejamento 4D. A escolha destes softwares se deu pelo domínio prévio por parte dos autores, bem como pelos mesmos se caracterizarem como os de maior representatividade de uso nos estudos apontados pelo mapeamento sistemático realizado por Carneiro, em quatro bases de dados [7].

A metodologia BIM foi utilizada na produção dos projetos de arquitetura, instalações elétricas, água fria, esgoto, modelagem estrutural e planejamento de demolição e reforma para uma residência existente de cerca de 75 metros quadrados de área construída.

Durante o fluxo de trabalho toda modelagem foi pensada em uma extração exata de quantitativos e no planejamento 4D, dessa forma foi escolhido o método de paredes cebola, que consiste em um recurso onde se modela as paredes e pisos em camadas que possibilitam uma maior precisão e controle da modelagem $3 \mathrm{D}$, e por isso exige uma maior dedicação e conhecimento do profissional.

É importante pontuar a necessidade de se compreender qual a finalidade de cada modelo, pois é através dessa variável que o profissional terá melhor fator de aproveitamento ao que é proposto [8].

De maneira geral aliou-se as ferramentas da tecnologia BIM ao contexto da residência existente com o objetivo de se identificar as vantagens, e também as principais dificuldades do processo. 


\section{Resultados}

Com os resultados encontrados durante o processo de modelagem 3D foi possível perceber a importância de se compreender a finalidade do modelo. Na Figura 2 é representada a modelagem do AS BUIT, ou como a residência se encontra no momento, e também exibe a proposta de reforma.
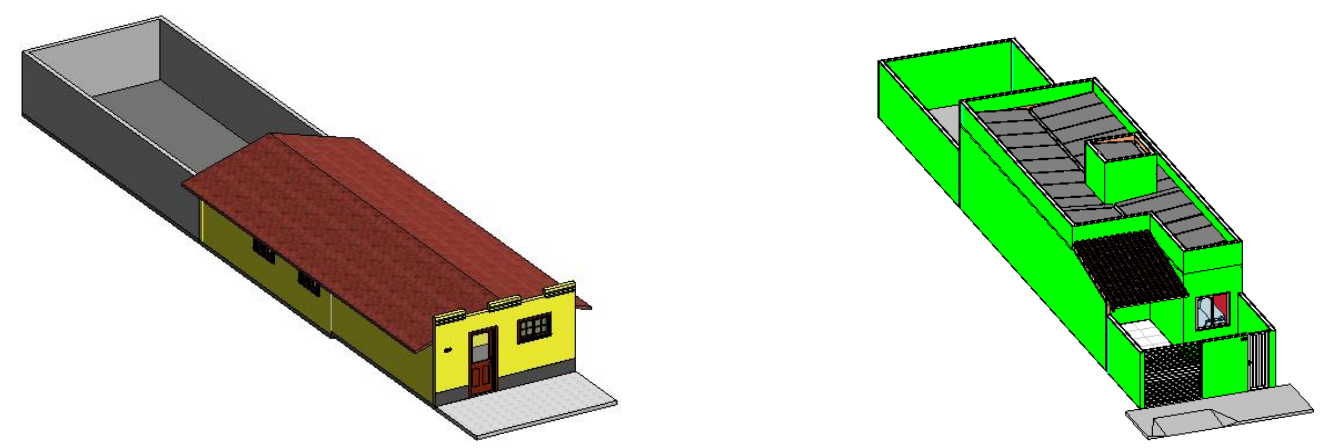

Figura 2: Modelagem AS BUIT, e modelagem para reforma.

Segundo Monteiro e Lima, o processo de implementação BIM começa com a definição da estratégia de integração da metodologia BIM nos processos de trabalho [9].

No caso da residência trabalhada foi evidenciado que para fins de levantamento dos quantitativos de materiais para demolição deveria ser realizada uma modelagem simples, de módulo único, já para a nova edificação seria necessária uma modelagem mais complexa, de forma a permitir o planejamento 4D em diferentes fases, ambos os casos são representados na Figura 3.
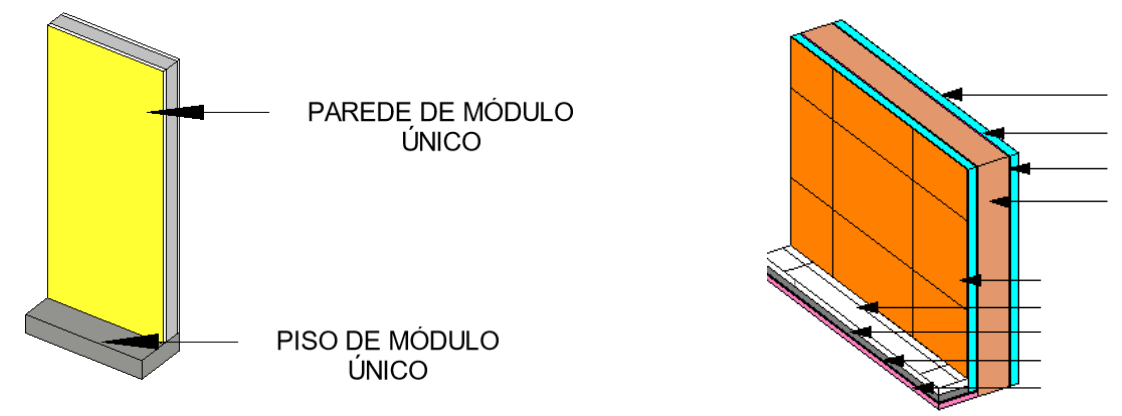

PINTURA

REBOCO/MASSA ÚNICA CHAPISCO

TIJOLO CERÂMICO

REVESTIMENTO CERÂMICO PISO CERAMICO CONTRAPISO AREA MOLHADA IMPERMEABILIZANTE REGULARIZAÇAOO

Figura 3: Modelagem módulo único, e modelagem em diferentes fases.

Durante a fase de extração de quantitativos foi possível constatar que quanto maior era o nível de detalhamento na modelagem, maior era o grau de precisão nos resultados apresentados nas tabelas.

No decorrer do processo também ficou evidente o fato extremamente relevante de se utilizar um Sistema de Classificação da Informação (SCI), ou seja, classificar de forma bastante organizada as camadas criadas, sejam elas para fins de revestimentos de paredes, pisos, ou demais elementos. 
Isso ocorre, pois, a metodologia de criar diversas camadas constituem um grande número de elementos, que quando são nomeados e bem classificados, através do SCI não causam problemas ao modelo geral. Nesse sentido, é notório que a eficiência do processo de modelagem da informação decorre de uma gestão efetiva da informação do projeto, o que é alcançado com a aplicação de um Sistema de Classificação da Informação [10].

Na Figura 4 descreve-se a situação em que os revestimentos coletados pelas tabelas não estavam com grau de modelagem elevado, e dessa forma ultrapassavam seus devidos locais de assentamento.

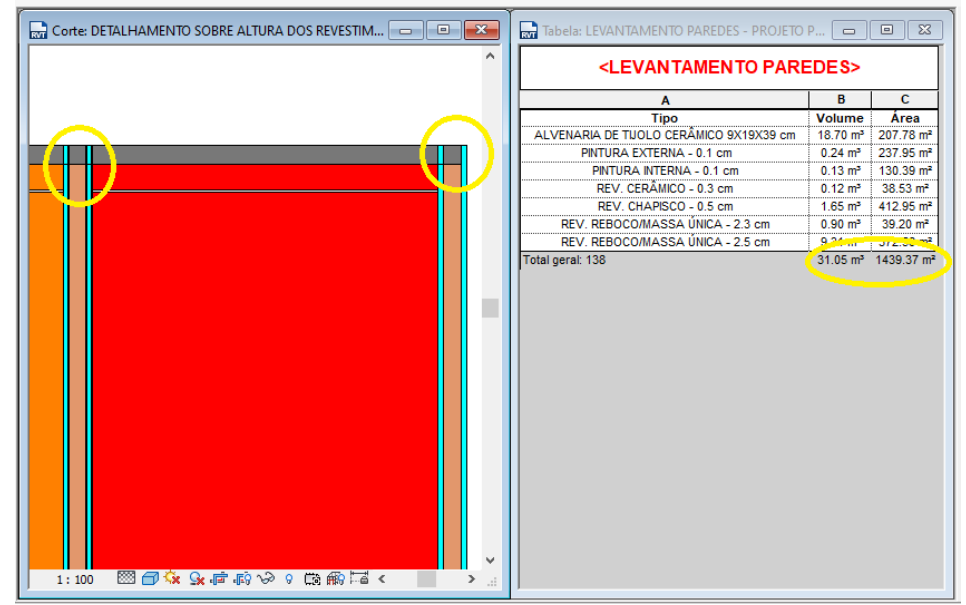

Figura 4: Modelagem parcial.

Na Figura 5 é possível observar que com uma modelagem de um grau de desenvolvimento maior foi possível obter uma extração de quantitativos mais precisa, e numericamente observou-se uma redução de cerca de $3 \%$ nos valores extraídos das superfícies em m².

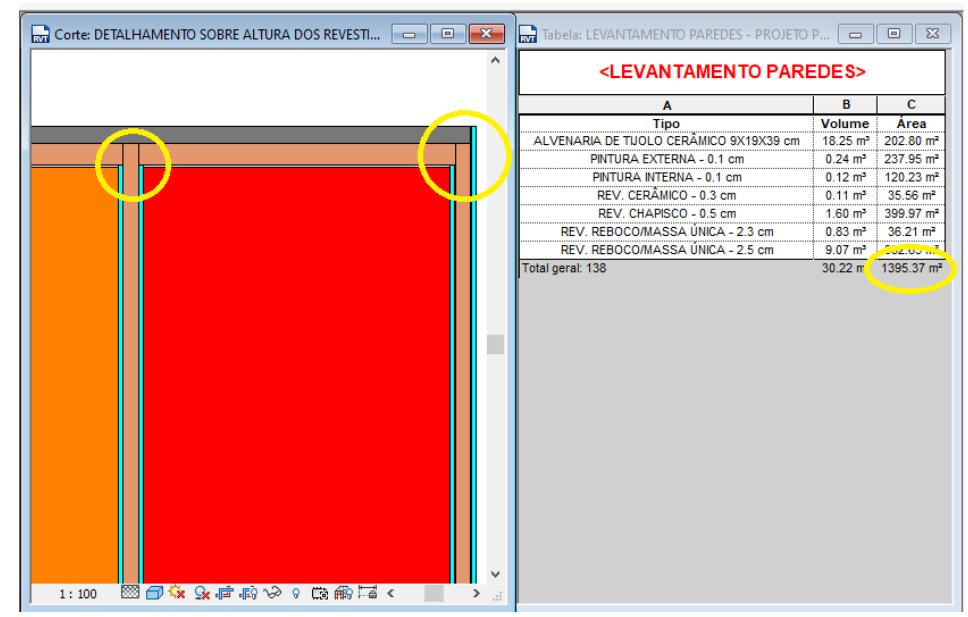

Figura 5: Modelagem finalizada.

A constatação da relação diretamente proporcional do nível de modelagem com a economia promovida ao empreendimento exibe benefícios tanto para uma edificação de pequeno porte, como gigantescos números para empreendimentos de grandes dimensões. 
Tratando-se da fase de compatibilização foi permitido antecipar problemas que só seriam detectados in loco, e dessa forma proporcionariam despesas aliadas ao retrabalho da mão de obra, aquisição de materiais e atraso significativo no cronograma físico-financeiro.

A aplicação da metodologia BIM promove: melhor comunicação entre as partes intervenientes; rapidez no processo de definição de uma nova solução; e previsão antecipada de incompatibilidades, permitindo estudar possíveis impactos nas diversas especialidades [11].

Através da Figura 6 é possível notar um dos momentos em que um elemento estrutural entraria em colisão com um componente das instalações elétricas, e por meio da visualização antecipada do problema em projeto foi possível executar a intervenção.
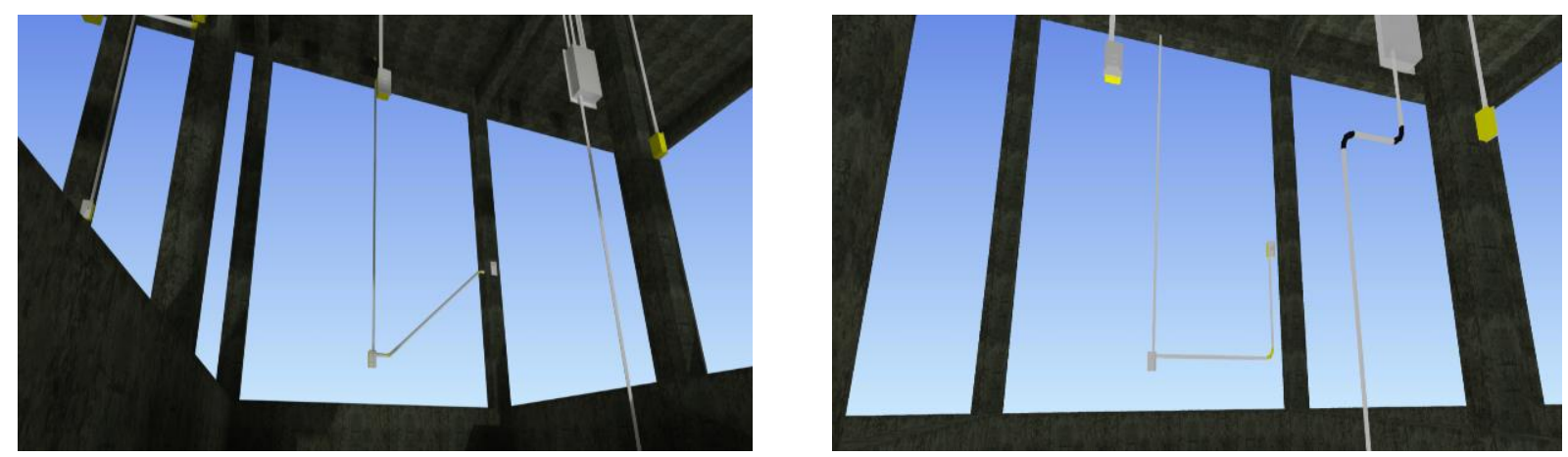

Figura 6: Conflito e intervenção entre componentes.

Um fato de grande relevância a ser relatado é sobre a interoperabilidade do fluxo do processo, foi possível observar dificuldades na interação com projetos que não apresentavam a mesma coordenada original que os demais.

Diante disso é perceptível que independente do software utilizado é de extrema importância que seja adotado um ponto base de projeto semelhante em todas as plataformas usadas. A Figura 7 mostra o momento em que é constatado a importância da coordenada base.

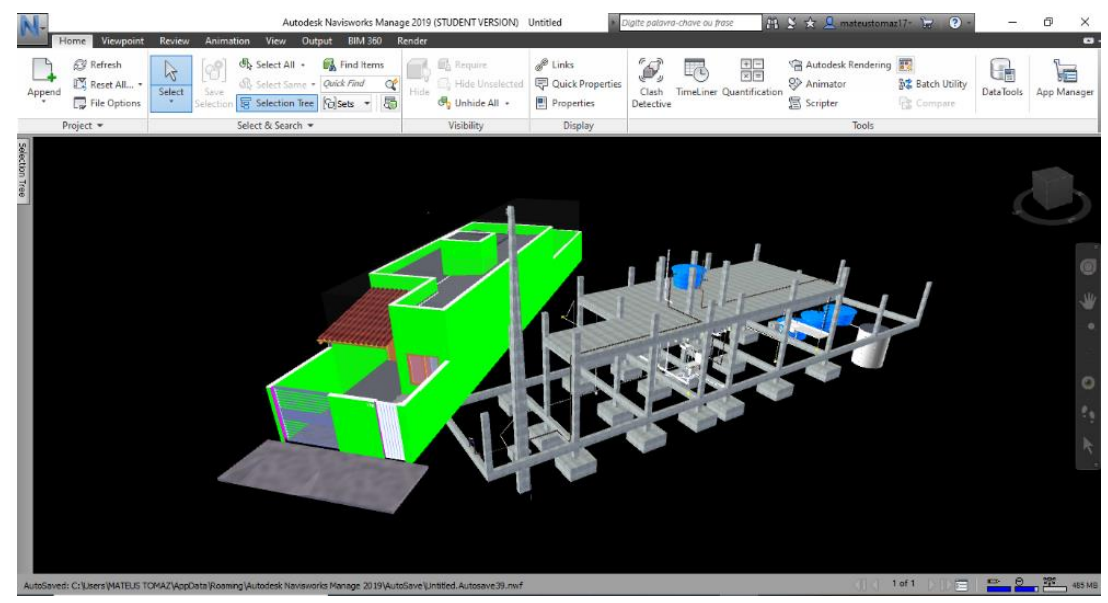

Figura 7: Interferência da coordenada base.

A dedicação na fase de projeto reflete na fase de construção da edificação, pois com um projeto bem feito, com disciplinas compatibilizadas, cronograma e quantitativos bem definidos obtémse um melhor trabalho durante a construção da obra. O retrabalho é menor, portanto, evita-se 
gastar dinheiro e tempo com decisões que normalmente seriam tomadas no canteiro de obra [12].

A Figura 8 exibe o conjunto de projetos já compatibilizados, é interessante relatar que durante a etapa de verificação de interferências surgem notificações de diversos pontos que não necessariamente representam uma colisão, dessa forma cabe ao profissional a interpretação de cada situação.

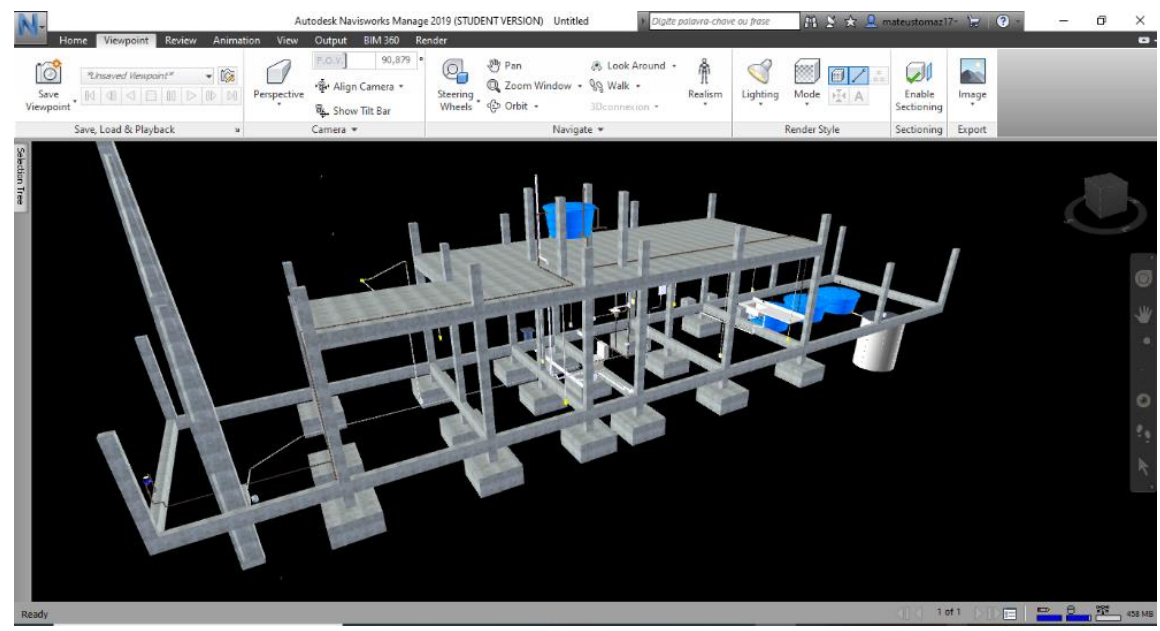

Figura 8: Projetos compatibilizados.

Uma ferramenta indispensável para o planejamento 4D foi a Estrutura Analítica do Projeto (EAP), ela pode ser vista na Figura 9.

Como o BIM 4D acrescenta a variável tempo, foi necessário que a EAP se apresentasse como o espelho da modelagem, assim os elementos 3D foram vinculados ao cronograma.

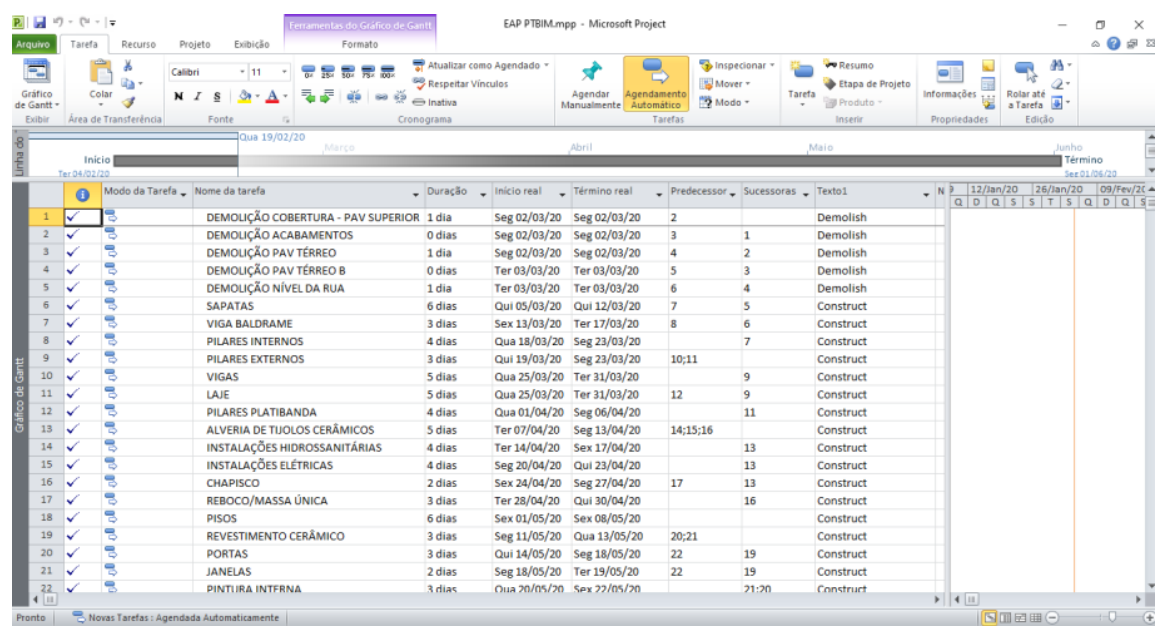

Figura 9: EAP.

De maneira global pode-se afirmar que o planejamento desenvolvido fornece ao mercado da construção civil uma visualização concreta do que ocorre nas fases da construção, podendo inclusive permitir a exibição de um plano de ação em caso de atrasos ou eventos inesperados durante a execução. 
A utilização da metodologia BIM forneceu a integralização de informações que só seriam visualizadas com a execução da construção, desta forma ao montar um planejamento que reflete a situação real da residência, que vai da sua demolição até a fase final da nova edificação, promove-se a entrega de um conjunto de benefícios que agregam além do fator econômico a variável de maior aproveitamento possível de tempo.

A interface mostrada na Figura 10 exibe a possibilidade de gerenciar a visualização das fases da edificação em qualquer período da obra.
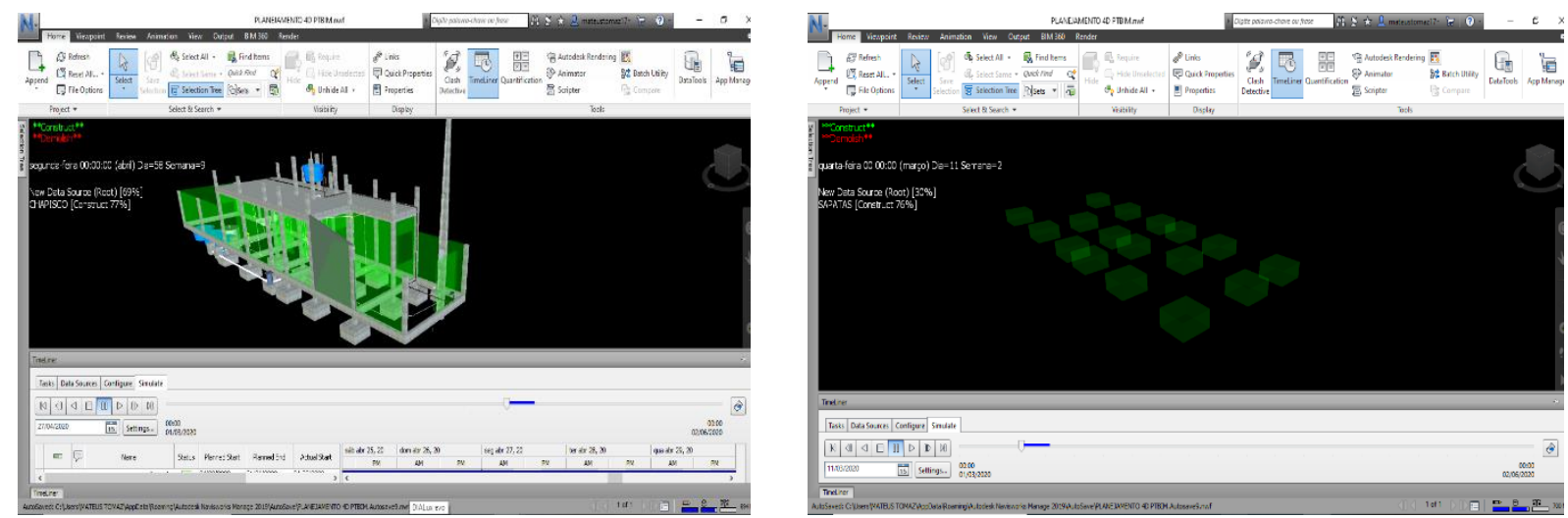

Figura 10: Planejamento 4D em diferentes fases da obra.

\section{Conclusão}

Todavia, ao final desse trabalho é possível concluir que a implementação da tecnologia BIM no processo construtivo é um evento indispensável quando se deseja obter racionalização e maior controle da gestão de obras.

Tendo em vista que a indústria da construção civil apresenta inúmeras inadimplências, com a apresentação de um escopo que mostra maior exatidão na indicação dos quantitativos e prazos especulados, promove-se um maior controle de qualidade na gestão dos processos.

O presente estudo fornece como principal contribuição a comprovação da viabilidade de implementação da tecnologia BIM, uma vez que possibilita maior otimização e segurança nos processos de extração de quantitativos e controle da visualização das diferentes fases da obra. Além de permitir a percepção antecipada de interferências que poderiam gerar retrabalhos durante a execução.

As adversidades encontradas no decorrer do estudo refletem para a necessidade de um planejamento não apenas dentro dos softwares utilizados, mas para uma avaliação previa do desenho de qual o melhor fluxo de trabalho, e quais programas apresentarão melhor eficiência junto ao objetivo buscado.

A presença de dificuldades durante o processo não inibe os resultados, apenas exibe o fato que quando se trabalha com mudanças dentro da metodologia de trabalho se torna natural a ocorrência de divergências até que se constatem os avanços. Diante disso e reconhecendo o apanhado de benefícios que a metodologia BIM pode proporcionar recomenda-se que esse estudo seja aplicado em conjunto da dimensão que agrega os custos (5D), pois com a adição da variável preço é possível promover ainda mais confiabilidade e precisão ao que é trabalhado. 


\section{Referências}

[1] J. Martins, "Modelação do Fluxo de Informação no Processo de Construção," Tese de Doutoramento, Faculdade de Engenharia da Universidade do Porto, 2009.

[2] C. Eastman, P. Teicholz, R. Sack and K. Liston, BIM Handbook, A Guide for Building Information Modeling for Owners, Managers, Designers, Engineers and Contractors, 2nd ed., New Jersey: John Wiley \& Sons, Inc., 2011.

[3] BRASIL, Decreto ${ }^{\circ}$ 9.983, de 22 de agosto de 2019. Dispõe sobre a Estratégia Nacional de Disseminação do Building Information Modeling e institui o Comitê Gestor da Estratégia do Building Information Modeling.

[4] BRASIL, Decreto $n^{\circ} 10.306$, de 02 de abril de 2020. Dispõe sobre a utilização do Building Information Modelling - BIM ou Modelagem da Informação da Construção na execução direta ou indireta de obras e serviços de engenharia, realizada pelos órgãos e pelas entidades da administração pública federal, no âmbito da Estratégia Nacional de Disseminação do Building Information Modelling - Estratégia BIM BR.

[5] J. C. Lino, N. Lacerda, F. Reis, B. Caires, "Soluções para a Coordenação, Implementação, e Gestão BIM - uma Perspectiva Global", in $1^{\circ}$ Congresso Português de Building Information Modeling (2016), Guimarães, Portugal, 2016, pp. 33-43. doi: 10.5281/zenodo.166758.

[6] D. Coelho, J. P. Couto, D. Leitão, J. Morgado, "Utilização do BIM 4D e 5D enquanto metodologia avançada para o planejamento, preparação e monitorização de obras", in $1^{o}$ Congresso Português de Building Information Modeling (2016), Guimarães, Portugal, 2016, pp. 437-449. doi: 10.5281/zenodo.166758.

[7] N. S. e R. Carneiro, "Panorama do uso do BIM 4D e 5D no planejamento e gerenciamento de obras na construção civil," TCC de Graduação em Engenharia Civil, Faculdade de Engenharia Civil, Universidade Federal de Uberlândia, Uberlândia, 2019.

[8] T. Gouveia, J. C. Lino, J. P. Couto, "Integração de ferramentas BIM aplicadas a um caso de estudo - Coordenação e quantificação", in $1^{\circ}$ Congresso Português de Building Information Modeling (2016), Guimarães, Portugal, 2016, pp. 409-418. doi: 10.5281/zenodo.166758.

[9] A. Monteiro, J. Lima, "Implementação de processos BIM: Desafios na aplicação da metodologia em diferentes enquadramentos da indústria da construção", in $2^{\circ}$ Congresso Português de Building Information Modeling (2018), Lisboa, Portugal, 2018, pp. 89-98. doi: 10.5281/zenodo.1226766.

[10]R. T. S. Martins, "Building Information Modeling: classificação de informação da construção para o planejamento e controle da produção," Monografia de Especialização, Curso de Engenharia Civil, Universidade Federal do Rio Grande do Sul, Porto Alegre, 2015.

[11] J. M. Silva, A. Calheiros, M. Azenha, "Digitalização da construção no Dstgroup: Implementação da metodologia BIM", in $2^{\circ}$ Congresso Português de Building Information Modeling (2018), Lisboa, Portugal, 2018, pp. 497-506. doi: 10.5281/zenodo.1226766.

[12] L. R. Steiner, R. R. Machado, “Análise da implementação da plataforma BIM no setor da AEC do estado de Santa Catarina", in $2^{\circ}$ Congresso Português de Building Information Modeling (2018), Lisboa, Portugal, 2018, pp. 19-28. doi: 10.5281/zenodo.1226766. 